\title{
Modelling and Analysis on Hot Rolling Process for Aluminum Plate Deformation based on Numerical Simulation
}

\author{
Jinxia Niu \\ Qinghai University, \\ Xining,Qinghai,810016 China
}

\author{
Jian Li \\ Qinghai University, \\ Xining,Qinghai,810016 China
}

\begin{abstract}
With the continuous development and advancement of mathematical modelling technique and manufacturing chain, the applications of combining mathematical simulation with production are well appreciated. Therefore, in this paper, we introduce research on hot rolling process for aluminum plate deformation based on numerical simulation. Heat transfer phenomenon in rolling process is very complex thermodynamic problems, rolled piece through the free surface and the surrounding medium heat, exist within the rolled piece and heat conduction, and still exist in the rolling process due to plastic deformation and friction heat. In the experiment part, we show the effectiveness of the method. Finally, we schedule future research and set up the prospect.
\end{abstract}

Keywords: Hot Rolling Process; Aluminum Plate Deformation; Numerical Simulation; Modelling.

\section{Introduction}

Aluminum clad alloys are commonly used because they allow to combine the individual advantages of different alloys concerning their physical and structure features. Rolling temperature is the important parameter in aluminum strip hot rolling process, it not only affects the deformation resistance of metal, plastic, and the size of the deforming force can parameters, and it is through the organizational structure of the metal after the impact rolling microstructures and properties of the product. The current computing temperature field in the process of rolling is an important means of modern material forecast. Heat transfer phenomenon in rolling process is very complex thermodynamic problems, rolled piece through the free surface and the surrounding medium heat, exist within the rolled piece and heat conduction, and still exist in the rolling process due to plastic deformation and friction heat. With the method of numerical simulation experiment is trend in theory study in recent years. Studies show that flat rolled piece hot rolling using two-dimensional conduction model approximation instead of 3D model is feasible. Generally, thermal conductivity of deposited thin films is different from their bulk form due to the special nature of the microstructure resulting from the growth process. The current methodology has the following drawbacks. (1) Previous subsection cooling control target for flatness of rolled piece. According to flatness gauge measured wave form characteristics of simple local cooling control, rather than from the root cause of the wave form which controlled the section shape of rolled piece, just as the remedy for product quality, not involved in the formation process of wave shape. (2) In view of each cooling section of segmented cooling system cooling water separate adjustable is not sufficient, the characteristics of the research can't provide effective guidance for practical production. (3) Thermal roll shape of work roll gap shape as an important component part of the study of the current confined to the steady state level, the previous studies did not give a quantitative relationship between the cooling water and the work roll thermal expansion, also does not 
change from the perspective of dynamic analysis of cooling water, work roll thermal expansion changes over time. Optimized content is on the premise of meet the process and equipment constraints, in order to reduce the total power consumption and improve the quality of strip. Therefore, related optimization based on simulation is urgently needed [1-2].

In this manuscript, we introduce research on hot rolling process for aluminum plate deformation based on numerical simulation. Aluminum hot rolling is a complicated system with nonlinearity, coupling and variable, predict the plate crown size can effectively improve the strip shape quality. Soft measurement technology can effectively solve the process quality indicators, which are hard to measure on-line estimation problem, and widely applied in the rolling process. Least squares support vector machine algorithm is based on support vector machine algorithm of the improved algorithm. It is based on small sample, to realize the structural risk minimization which can approximate arbitrary precision of nonlinear system which is a powerful tool for nonlinear system modeling [3]. We will introduce the related numerical simulation and theoretical analysis in the following parts. In the figure one, we illustrate the structure of the general system for analyzing.

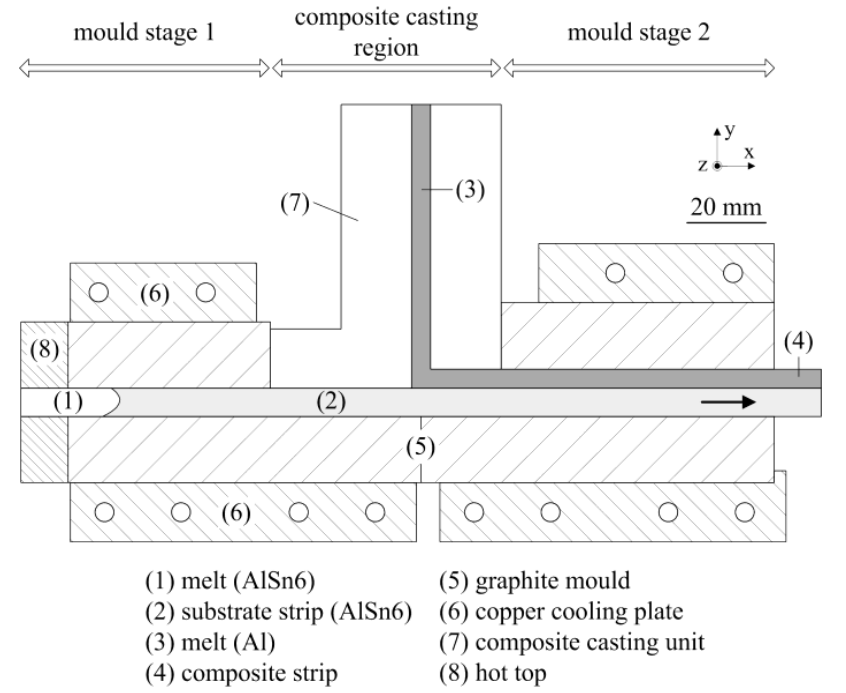

Fig. 1The General System Structure for Processing

\section{The Proposed Approach}

The Least Squares Support Vector Machine. Least squares support vector machine using the least squares linear system as the loss function, the inequality constraints into equality constraints which become to understand a set of equations solving process, speed relatively. When continuous sliding phenomenon directly affect the quality of the surface of aluminum strip, serious when a scratch, product scrap, economy loss could not be ignored [4]. By the field experience and research, the board with a scratch is due to skid when hot rolling, in aluminum hot rolling skid phenomenon, mainly is the strip deformation zone completely replaced by the slip zone after. The optimization process for the machine could be formulated as the formula one.

$$
\left\{\begin{array}{c}
\min _{w, e} J(w, e)=\frac{1}{2} w^{T} w+\frac{1}{2} \xi \sum_{i=1}^{N} e_{i}^{2} \\
\text { s.t. } \quad y_{i}=w^{T} \varphi\left(x_{i}\right)+b+e_{i}
\end{array}\right.
$$

(1)

About aluminium strip rolling mill's different characteristic parameters, aluminum can bring along the width direction of the force parameters of unequal distribution factors often cause aluminium rolling system set the centerline deviation from the center line, running deviation phenomenon. Aluminum belt run destroy the stability of the rolling process, led to the suspension of operation failure, lead to the production process [5]. The core factors are shown in the formula $2 \sim 3$.

$$
\begin{aligned}
& \alpha=\arccos (1-\sqrt{\Delta h / 2 R}) \\
& \gamma=\sqrt{\frac{h_{i-1}}{R}} \tan \left[\frac{1}{2} \operatorname{artan} \sqrt{\frac{\varepsilon}{1-\varepsilon}}+\frac{\pi}{8} \ln (1-\varepsilon) \sqrt{\frac{h_{i-1}}{R}}\right]
\end{aligned}
$$

(3)

At present, the commonly used optimization method for parameters include: cross validation method, genetic algorithm and particle swarm optimization algorithm, etc. Optimization efficiency is low, the algorithm easily fall into 
local optimum, which affect the precision and generalization performance of the whole model. Artificial colony algorithm is a kind of new optimization algorithm simulation of the colony behavior. In the algorithm, colony by bees, follow three bee bee, bee budgeting. Colony individuals through division of labor cooperation and information transfer to obtain the optimal solution. Food source location on behalf of the optimization problem of feasible solution, how much the amount of nectar on behalf of the size of the fitness. When a food source is followed by bees and bee abandon, this food source for the corresponding bees becomes scouts. The description of the aalforithm is shown in the formula 4 .

$$
x_{i}=x_{\text {min }}+\operatorname{rand} \cdot\left(x_{\max }-x_{\text {min }}\right)
$$

In the later process of numerical simulation, we will adopt the algorithm.

The Structure of Hot Rolling Process. Under the action of rolling force, deformation of rolled piece, its overall dimensions and cross section shape change. Reaction of rolled piece also makes the work of mill stand produce elastic deformation. Usually will be the sum of the elastic deformation of mill stand work called jumping value of the rolling mill. Under the action of rolling force, work stand produce elastic deformation, the thickness of the rolled piece is greater than the initial values of roll, and uneven distribution of the thickness of the rolled piece width direction.

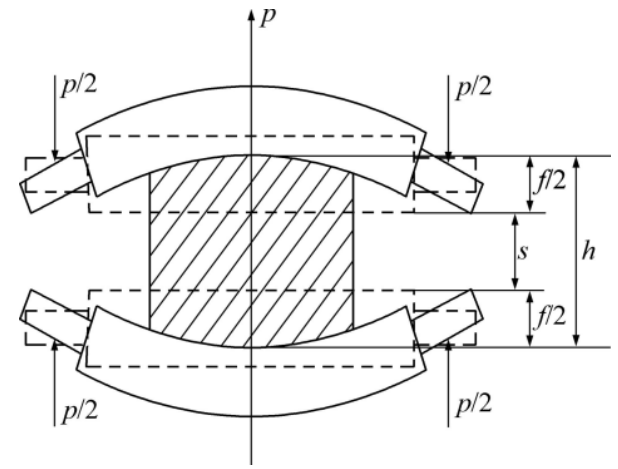

Fig. 2The General Structure of the Proposed System

Plate thickness precision of the rolled piece is one of the important quality indexes of rolled pieces and the essence of the thickness control is carried out on the mill roll gap control. The thickness of the traditional prediction methods is through pressure by method or the measure methods of rolling mill under different rolling force value bounce, bounce curve measured by mill stand and then establish the mathematical model of stand bounce curve. Due to ignore in the process of the establishment of the mathematical model and simplified many factors, therefore, to calculate the thickness of the error is bigger, cannot satisfy the increasing needs of the people. The solidification length is decided by the position of a nozzle-plate which is shown in the figure 3.

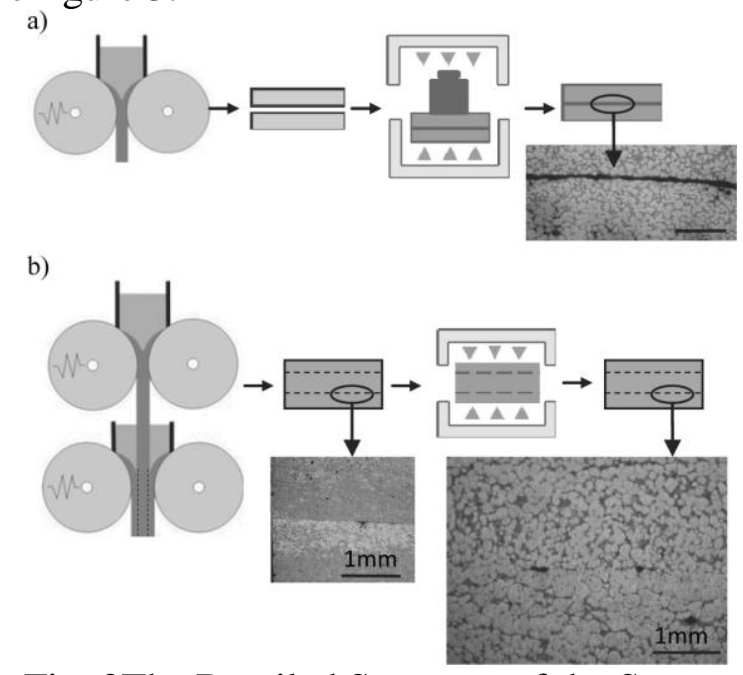

Fig. 3The Detailed Structure of the System

The Prior Steps before Simulation. In order to simplify the network, improve the speed of learning and training, in the input parameters above does not include the mill work roll diameter, backup roll diameter, rolling mill stiffness, roll crown and factors such as emulsion flow changes because of their relatively stable. Training sample selection of typical and practical problems needs to pay attention to its own characteristics, a reasonable choice. This article selects the method is: first of all, removing a few error significantly larger data, then adopt the method of equidistance sample selection. The width coefficient has more influence on the performance of the network. The width coefficient, the greater the network approximation process of sample data is more 
smooth, however, approximation error is larger; Width coefficient is smaller, the approximation properties of the samples is better, but the approximation process is not smooth, and over fitting phenomenon may occur. In the following section, we will show the result of simulation and experiment.

\section{Experiment and Numerical Simulation}

In this section, we take research on the simulation of the proposed methodology. The sound three layers clad strip, which base strip has lower melting than that of the overlay strip, could not be cast by the vertical type tandem twin roll caster. In the figure 4, we illustrate the numerical simulation result for the system. We could conclude that our method outperforms the others.

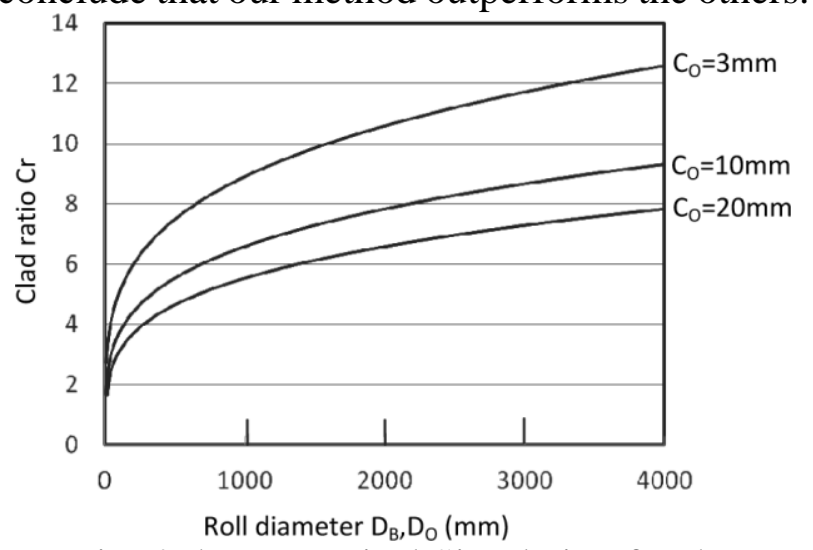

Fig. 4The Numerical Simulation for the

Proposed Method

\section{Conclusion}

In this paper, we introduce research on hot rolling process for aluminum plate deformation based on numerical simulation. Least squares support vector machine algorithm is based on support vector machine algorithm of the improved algorithm. It is essential to cast sound clad strip that the melt of the alloy of the lower melting point contacts to the solidification layer of the alloy of higher melting point. It is based on small sample, to realize the structural risk minimization which can approximate arbitrary precision of nonlinear system. Combining the numerical simulation and the real-world organization of the system, we could achieve better result and performance of the manufacturing process. In the future, we plan to conduct more related research to polish the current method.

\section{Acknowledgement}

Fund project: the department of science of qinghai province' $\mathrm{s}$ project $<$ Numeirical simulation and process optimization of hot-rolling on industrial aluminum plate >Item number: (2013-G-Q15A-1)

\section{References}

[1] Yulan, L., Yilun, L., Wei, Z., \& Liyong, M. (2014). Deviation control research for hot rolled aluminum plate based on fuzzy theory. Forging \& Stamping Technology, 39, 3, 137-140.

[2] Xizhao, Z., Yunxin, W., Tao, Z., \& Hai, G. (2014). Effect of dragon rolling parameters on shear deformation in center of aluminum alloy thick plate. Hot Working Technology.

[3] Chang-qing, H., Bin, L., Min, Z., \& Kang, Y. (2014). Optimization of hot finishing rolling schedule for aluminum strip based on improved genetic algorithm. Metal Materials $\&$ Metallurgy Engineering.

[4] Yanjuan, J., Xiaochao, C., \& Zhu, Z. (2014). Numerical simulation of cast-rolling process for strip of fiber reinforced aluminum matrix composites. Foundry Technology.

[5] Chen, S., Da-heng, M., \& Chang-qing, H. (2014). Effect of electromagnetic-ultrasonic energy field on recrystallization microstructure and texture of 1060 aluminum alloy plate. Chinese Journal of Nonferrous
24
3 ,
606-614. 